\title{
Effects of Mild and Severe Knee Joint Pain on the Frequency of Falls and Fall Risk in Elderly Females
}

\author{
Hiroki Sugiura ${ }^{*}$, Shinichi Demura ${ }^{2}$ \\ ${ }^{1}$ Department of Industrial Business and Engineering, Fukui University of Technology, Fukui, Japan \\ ${ }^{2}$ Graduate School of Natural Science and Technology, Kanazawa University, Ishikawa, Japan \\ Email: sugiura@fukui-ut.ac.jp
}

Received 15 July 2014; revised 20 August 2014; accepted 17 October 2014

Copyright (C) 2014 by authors and Scientific Research Publishing Inc.

This work is licensed under the Creative Commons Attribution International License (CC BY). http://creativecommons.org/licenses/by/4.0/

(c) (7) Open Access

\section{Abstract}

This study aimed to examine differences in the frequency of falls during the past year and fall risk among 392 elderly females was categorized into five groups on the basis of the knee pain experienced: no knee pain, mild unilateral knee pain, mild bilateral knee pain, severe unilateral knee pain, and severe bilateral knee pain. The subjects participated in a fall risk survey comprising $\mathbf{5 0}$ items representing five risk factors: "symptoms of falling", "disease and physical symptoms", "environment", "behavior and character", and "physical function". The frequency of falls during the past year, score for each risk factor, and the total fall risk score were not significantly different between the mild unilateral and mild bilateral knee pain groups, and between the severe unilateral and severe bilateral knee pain groups. Therefore, these groups were pooled to form a mild knee pain group and a severe knee pain group and analyzed. The severe knee pain group had experienced a significantly greater number of falls during the past year compared with the no knee pain group. Furthermore, the symptom of falling score was significantly higher in the severe knee pain group than in the no knee pain group, while the disease and physical symptoms score was significantly higher in the mild and severe knee pain groups than in the no knee pain group. The physical function score and total fall risk score were significantly higher in the following order: the severe knee pain group, the mild knee pain group, and the no knee pain group. Our results indicate that for elderly females who can achieve ADL independently, the degree of knee pain (mild or severe) has a marked effect on fall risk, irrespective of laterality of the pain (unilateral or bilateral). Factors such as symptoms of falling, disease and physical symptoms, and physical function are also related to fall risk in this population. Furthermore, elderly individuals with severe knee pain experience frequent falls.

\footnotetext{
${ }^{*}$ Corresponding author.
} 


\section{Keywords}

Mild Knee Pain, Severe Knee Pain, Frequency of Falls, Fall Risk, Elderly Females

\section{Introduction}

With advancing age, physical functions, such as leg strength, balance, and leg joint function, show a marked decrease, thus limiting activities of daily living (ADL) [1] [2]. A decrease in ADL in turn influences fall risk [3] [4]. Therefore, to avoid falls, it is very important to prevent a decrease in the ability to achieve ADL [5] [6].

Advanced lower limb arthritis often develops in the knee joints [7] and increases the fall risk [8]. Among all the lower limb joints, the knee joint has the greatest load-bearing capacity [9]; therefore, any impairment in this joint makes it difficult to walk or stand up, which represents the most basic physical ADL. However, there are individual differences in the degree of pain and severity of the condition among the elderly with knee joint pain. Tennant et al. [10] reported that $8 \%$ of elderly individuals had such severe knee pain that they require regular visits to a hospital or hospitalization. On the other hand, elderly individuals with mild knee pain who do not require regular visits to a hospital are considered to be an auxiliary group of the severe knee pain group [7] [11] [12] and account for approximately $20 \%$ of the elderly population [13]-[15]. In addition, elderly individuals with knee pain are divided into those with unilateral knee pain and bilateral knee pain. These groups may have different fall risks. Although many studies have focused on elderly individuals with severe pain [12] [16] [17], few have examined the frequency of falls and fall risk among elderly individuals with knee pain, including mild knee pain. Moreover, it has been reported that elderly females have a high prevalence of knee pain [7] [18].

This study aimed to examine differences in fall experiences during the past year and fall risk among elderly females without knee pain, mild unilateral or bilateral knee pain, and severe unilateral or bilateral knee pain.

\section{Method}

\subsection{Participants}

Even if the elderly have orthopedic abnormalities, approximately 50\% do not feel subjective knee pain [7] [19]. Peat et al. [7] reported that it is necessary to focus on pain in the knee joints because it is common among the elderly and varies in type and cause. In this study, elderly individuals who selected Yes in response to the question "Do you have an articular disorder (ankle, knee, hip joint)? (choice: Yes and No)" and Right, Left, or Both in response to the question "Do you have knee pain or disorders? (choice: Right, Left, Both, and No)" were defined as patients with knee pain. Individuals who selected No in response to both questions were defined as those without knee pain. Mild and severe knee pain was judged using the Japanese edition knee function scale [20] on the basis of the Western Ontario and McMaster Universities Osteoarthritis Index [21]. According to this assessment, individuals with over 210 points and those with less than 210 points were judged to have severe knee pain and mild knee pain, respectively [18].

After the above survey was administered to 1280 adults [age range, 29 - 94 years; mean age, 72.6 years; standard deviation (SD), 8.7 years], 392 elderly females (age range, 60 - 94 years; mean age, 72.8 years; SD, 6.8 years) were selected as participants (Investigation area: Fukui, Japan. Investigation period: 2012.8 - 2013.9). These included 225 without knee pain [no knee pain (G1) group], 71 with mild unilateral knee pain (G2 group), 35 with mild bilateral knee pain (G3 group), 34 with severe unilateral knee pain (G4), and 27 with severe bilateral knee pain (G5 group). The participants attended health classes or social educational activities hosted by municipal governments and engaged in social activities at least once per week or every alternate week. In short, they could independently perform ADL. The purpose and procedures of this study were explained in detail to all participants before their informed consent was obtained. The present experimental protocol was approved by the Ethics Committee on Human Experimentation of Faculty of Human Science, Kanazawa University (Ref. No. 2012-12).

\subsection{Fall Risk Assessment}

Demura's fall risk assessment [22] was used in this study (Table 1). This comprised 50 items representing the 
Table 1. Demura's fall risk assessment.

\begin{tabular}{|c|c|c|}
\hline Risk factors & & Question \\
\hline \multirow{3}{*}{ Symptoms of falling ( 3 items) } & 1. & In the past year, have you felt like you might fall down? \\
\hline & 2. & Have you often stumbled? \\
\hline & 3. & Have you ever been told that you look like you might fall down? \\
\hline \multirow{13}{*}{$\begin{array}{c}\text { Disease and physical symptoms (13 } \\
\text { items) }\end{array}$} & 4. & Do you ever feel lightheaded upon standing up? \\
\hline & 5. & Do you ever feel dizzy upon standing up? \\
\hline & 6. & Are you taking any medications, daily? \\
\hline & 7. & Have you ever had a circulatory disease? \\
\hline & 8. & Do you feel forgetful these days? \\
\hline & 9. & Can you hear well (people talking, etc.)? \\
\hline & 10. & Can you see well (newspaper, people's faces, etc.)? \\
\hline & 11. & Do you ever feel groggy? \\
\hline & 12. & Have you ever had a stroke? \\
\hline & 13. & Do you have an articular disorder (ankle, knee, hip joint)? \\
\hline & 14. & Do you have osteoporosis? \\
\hline & 15. & Have you ever had complications from a disease? \\
\hline & 16. & Have you ever been diagnosed as having diabetes? \\
\hline \multirow{4}{*}{ Environment (4 items) } & 17. & Are there slippery places in your house? \\
\hline & 18. & Are there obstacles that may cause someone to stumble in your house? \\
\hline & 19. & Do you wear sandals or slippers a lot every day? \\
\hline & 20. & Do your shoes fit your feet? \\
\hline \multirow{8}{*}{ Behavior and character ( 8 items) } & 21. & Do you often sit at home? \\
\hline & 22. & Do you hardly ever have occasions to go out? \\
\hline & 23. & Do you have many occasions to go to the toilet at night? \\
\hline & 24. & Do you act cautiously? \\
\hline & 25. & Are you confident about not falling? \\
\hline & 26. & Do you worry about falling? \\
\hline & 27. & Do you often climb up the steep slope? \\
\hline & 28. & Do you often rush about? \\
\hline \multirow{8}{*}{ Physical function (22 items) } & 29. & Are you strong enough to wring out a wet towel or cloth effectively? \\
\hline & 30. & Can you stand on one foot and put a sock on the other foot? \\
\hline & 31. & Can you stand on one foot about $5 \mathrm{~s} ?$ \\
\hline & 32. & Do you usually use walking aids such as stick or walker? \\
\hline & 33. & Do you feel your length of stride decrease? \\
\hline & 34. & Do you feel your walking speed becoming slower? \\
\hline & 35. & Can you walk continuously for about $1 \mathrm{~km}$ ? \\
\hline & 36. & Can you fold up and down a heavy futon? \\
\hline
\end{tabular}




\section{Continued}

\begin{tabular}{|c|c|c|}
\hline \multirow{14}{*}{ Physical function (22 items) } & 37. & Can you sit-up about 1-2 times? \\
\hline & 38. & Can you jump about a $30 \mathrm{~cm}$ gap? \\
\hline & 39. & Can you jump about a $50 \mathrm{~cm}$ gap? \\
\hline & 40. & Can you balance on one foot with open eyes for $30 \mathrm{~s}$ or more? \\
\hline & 41. & Can you stand on the bus or train without holding onto a hand strap or rail? \\
\hline & 42. & Can you walk about 60 min? \\
\hline & 43. & Can you run about 3 - $5 \min ?$ \\
\hline & 44. & Can you climb up stairs slowly without a handrail or wall for support? \\
\hline & 45. & Can you climb up stairs without a handrail and wall for support? \\
\hline & 46. & Can you stand from a sitting posture (Seiza) with your hands on the floor? \\
\hline & 47. & Can you stand from a sitting posture (Seiza) without using your hands? \\
\hline & 48. & Can you button or unbutton a shirt quickly with both hands? \\
\hline & 49. & Can you button or unbutton a shirt with single hand? \\
\hline & 50. & Can you carry a object weighing about $5 \mathrm{~kg}$ ? \\
\hline
\end{tabular}

following five risk factors: "symptoms of falling" (3 items), "disease and physical symptoms" (13 items), "environment" (4 items), "behavior and character" ( 8 items), and "physical function" (22 items). All responses were on a dichotomous scale (Yes or No), with one point being assigned to each response that fell into the high-risk category. The scores for each risk factor and the subject's total fall risk score were used as evaluation parameters. A higher score was interpreted as a higher fall risk.

\subsection{Statistical Analysis}

Mean differences in each parameter were examined by ANCOVA using age as a covariate. Scheffe's test was used as a linear comparison test if a significant difference among mean values was found. The significance level was set at $p<0.05$ and was adjusted by Scheffe's method.

\section{Results}

Table 2 shows the basic age statistics, Table 3 shows the basic statistics and results of fall frequencies during the past year for the five groups, and Table 4 shows the basic statistics and scores for each risk factor as well as the total fall risk score for the five groups. It was observed that mean age, fall frequency, scores for each risk factor, and the total fall risk score were not significantly different between the mild unilateral knee pain (G2) and mild bilateral knee pain (G3) groups and between the severe unilateral knee pain (G4) and severe bilateral knee pain (G5) groups. Therefore, these groups were pooled to form a mild knee pain group (G2 + G3) group and a severe knee pain (G4 + G5 group) and analyzed. One-way ANOVA revealed a significant difference in mean age among groups. Patients in the severe knee pain group (G4 + G5) were significantly older than those in the no knee pain and mild knee pain groups (G2 + G3).

Furthermore, ANCOVA revealed a significant difference in the fall frequency among groups. A linear comparison test showed that the G4 + G5 group experienced a significantly greater number of falls compared with the G1 group. ANCOVA also revealed a significant difference in "symptoms of falling", "disease and physical symptoms", and "physical function" scores as well as the total fall risk score among groups. A linear comparison test showed that the symptoms of falling score was significantly higher in the G4 + G5 group than in the G1 group, whereas the disease and physical symptom score was significantly higher in the G2 + G3 and G4 + G5 groups than in the G1 group. The physical function score and total score were significantly higher in the following order: G4 + G5 group, G2 + G3 group, and G1 group. 
Table 2. Basic statistics of age in the 5 groups and the test results.

\begin{tabular}{|c|c|c|c|c|c|c|c|c|c|c|c|}
\hline & & & & & & & \multicolumn{3}{|c|}{ ANOVA } & \multicolumn{2}{|c|}{ Scheff's Post-hoc } \\
\hline & & G1 & $\mathrm{G} 2$ & G3 & G4 & G5 & $\mathrm{F}$ & $\eta^{2}$ & $p$ & $\mathrm{G} 1, \mathrm{G} 2, \mathrm{G} 3, \mathrm{G} 4, \mathrm{G} 5$ & $\mathrm{G} 1,(\mathrm{G} 2+\mathrm{G} 3),(\mathrm{G} 4+\mathrm{G} 5)$ \\
\hline \multirow{4}{*}{ Age (yr) } & M & 71.5 & 73.0 & 73.4 & 76.2 & 77.6 & \multirow{4}{*}{$8.21^{*}$} & \multirow{4}{*}{0.08} & \multirow{4}{*}{0.00} & \multirow{4}{*}{$\begin{array}{c}\mathrm{G} 1, \mathrm{G} 2<\mathrm{G} 4, \mathrm{G} 5 \\
\mathrm{G} 3<\mathrm{G} 5\end{array}$} & \multirow{4}{*}{$\mathrm{G} 1, \mathrm{G} 2+\mathrm{G} 3<\mathrm{G} 4+\mathrm{G} 5$} \\
\hline & SD & 6.3 & 6.9 & 7.2 & 5.5 & 6.8 & & & & & \\
\hline & MAX & 87 & 94 & 86 & 87 & 87 & & & & & \\
\hline & MIN & 60 & 60 & 60 & 61 & 66 & & & & & \\
\hline
\end{tabular}

Note: G1: No knee pain ( $\mathrm{n}=225)$; G2: Mild unilateral knee pain $(\mathrm{n}=71)$; G3: Mild bilateral knee pain $(\mathrm{n}=35)$; G4: Severe unilateral knee pain $(\mathrm{n}=$ 34); G5: Severe bilateral knee pain $(\mathrm{n}=27) ;{ }^{*} p<0.05$.

Table 3. Basic statistics of fall frequencies during the past year in the 5 groups and the test results.

\begin{tabular}{|c|c|c|c|c|c|c|c|c|c|c|c|}
\hline & \multirow{2}{*}{ G1 } & \multirow{2}{*}{ G2 } & \multirow{2}{*}{ G3 } & \multirow{2}{*}{ G4 } & \multirow{2}{*}{ G5 } & \multirow{2}{*}{$\chi^{2}$} & \multirow{2}{*}{$p$} & \multirow{2}{*}{$\mathrm{G} 2+\mathrm{G} 3$} & \multirow{2}{*}{$\mathrm{G} 4+\mathrm{G} 5$} & \multicolumn{2}{|c|}{ Scheff's Post-hoc } \\
\hline & & & & & & & & & & $\mathrm{G} 1, \mathrm{G} 2, \mathrm{G} 3, \mathrm{G} 4, \mathrm{G} 5$ & $\mathrm{G} 1,(\mathrm{G} 2+\mathrm{G} 3),(\mathrm{G} 4+\mathrm{G} 5)$ \\
\hline Frequency of falls & 0 & 0 & 0 & 0.5 & 1 & $22.9^{*}$ & 0.00 & 0 & 1 & $\begin{array}{c}\mathrm{G} 1<\mathrm{G} 4, \mathrm{G} 5 \\
\mathrm{G} 2<\mathrm{G} 5\end{array}$ & $\mathrm{G} 1<\mathrm{G} 4+\mathrm{G} 5$ \\
\hline
\end{tabular}

Note: G1: No knee pain ( $\mathrm{n}=225)$; G2: Mild unilateral knee pain $(\mathrm{n}=71)$; G3: Mild bilateral knee pain $(\mathrm{n}=35)$; G4: Severe unilateral knee pain $(\mathrm{n}=$ 34); G5: Severe bilateral knee pain $(\mathrm{n}=27) ;{ }^{*} p<0.05$.

\section{Discussion}

In this study, patients with unilateral or bilateral severe knee pain were older than those without knee pain. In an old age period, the functions of the locomotorium, including the knee joints, decrease markedly as age increases. The knee joints play an important role in supporting body weight [23]. Therefore, it is considered that osteoarthritis and arthritis occur because of an increased burden of body weight on the knee joints due to a decrease in leg muscle and bone density with age. Increasing strength around the knee joints by training and exercise that do not impose excessive burden on the knee joints is useful as a precaution to prevent the onset or exacerbation of knee pain [24]; therefore, it is desirable, particularly for elderly individuals with severe knee pain, to rigorously engage in such training and/or exercise.

The fall risk assessment used here [22] includes five risk factors and synthetically evaluates the risk of falling. The total fall risk score and the score for each risk factor were not significantly different between the mild unilateral and mild bilateral knee pain groups and between the severe unilateral and severe bilateral knee pain groups. Furthermore, although it was hypothesized that patients with bilateral knee pain would be at an increased risk of falling compared to patients with unilateral knee pain, this hypothesis was rejected. According to Mary and Tinetti [4], a close relationship was found between walking ability and fall. Sugiura and Demura [23] reported that there was no significant difference in gait between the unilateral knee pain and bilateral knee pain groups in their study. From the present results, it appears that there is no significant difference in fall risk between elderly patients with unilateral knee pain and those with bilateral knee pain, irrespective of the degree of pain.

When the groups were unified into a mild knee pain group and a severe knee group and analyzed, it was found that the number of falls experienced during the past year was significantly greater in the severe knee pain group than in the no knee pain group, whereas it was comparable between the mild knee pain group and the no knee pain group. A limitation of this study was that falls from all causes, including knee pain and accidents, were included in this study. Therefore, it is not necessary adequately that all individuals with knee pain fell because of knee pain. However, despite the various causes, it was found that the participants with severe knee pain experienced a greater number of falls during the past year. This population is also considered to lack the ability to avert falls because of decreased knee extension strength [16] [23].

The total fall risk score in our study was significantly higher in the following order: the severe knee pain group, the mild knee pain group, and the no knee pain group. Many factors influence fall risk. There are large individual differences in the role played by each factor; furthermore, a different combination of factors may be 
Table 4. Basic statistics of fall risk scores in the 5 groups and the test results.

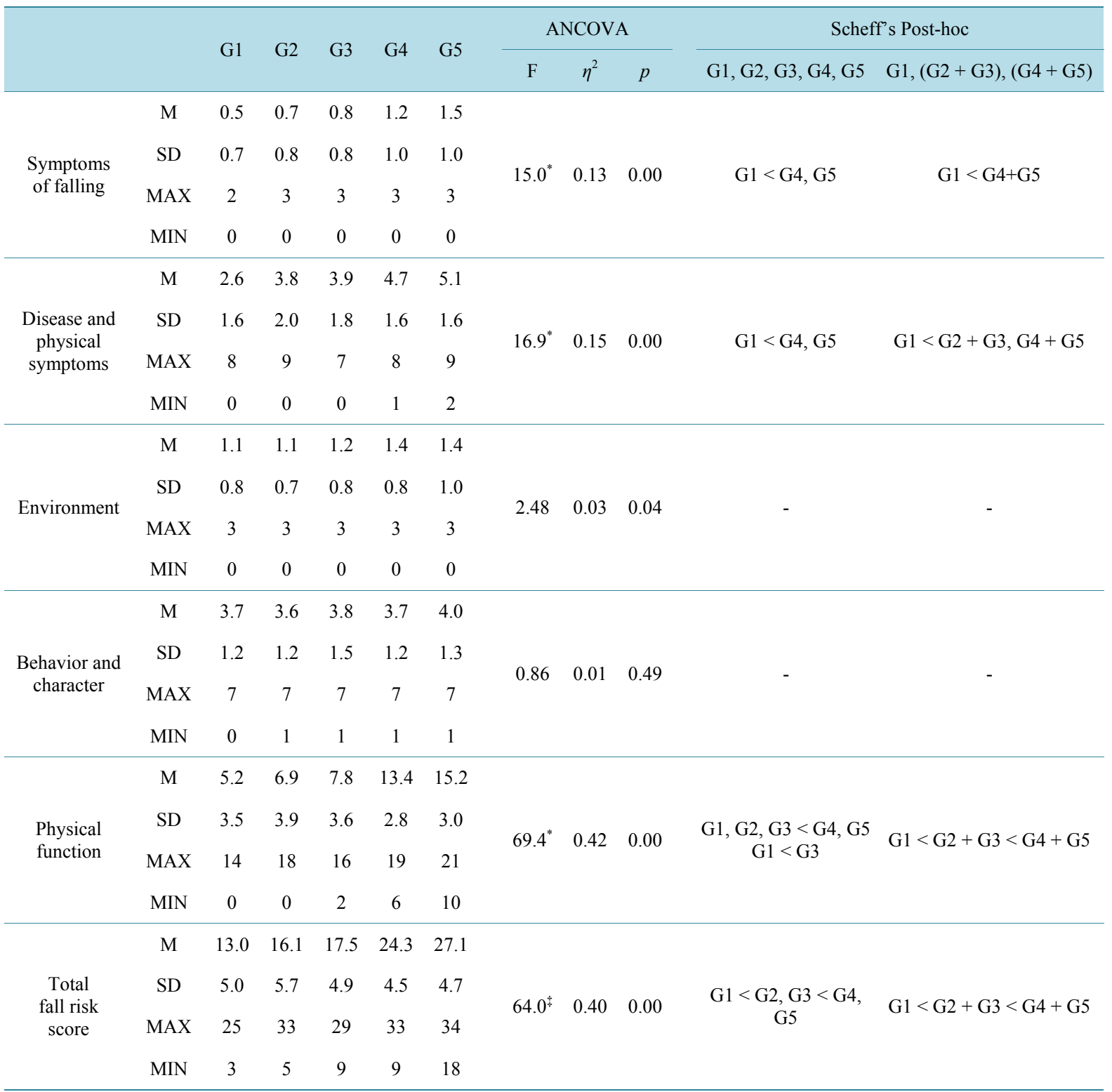

Note: G1: No knee pain $(\mathrm{n}=225)$; G2: Mild unilateral knee pain $(\mathrm{n}=71)$; G3: Mild bilateral knee pain $(\mathrm{n}=35)$; G4: Severe unilateral knee pain $(\mathrm{n}=$ 34); G5: Severe bilateral knee pain $(\mathrm{n}=27) ;{ }^{*} p<0.05 / 5=0.01 ;{ }^{\ddagger} p<0.05$.

present in individuals [8]. Therefore, it cannot be asserted that only knee pain affects fall. Our results showed that the number of falls was not significantly different between the mild knee pain group and the no knee pain group, although the risk of falling was higher in the former than in the latter. Patients in the mild knee pain group demonstrated superior ability to avert falls compared with patients in the severe knee pain group. However, elderly individuals with mild knee pain may have high fall risk and experience more falls in the future compared with those without knee pain.

The symptoms of falling score was significantly higher in the severe knee pain group than in the no knee pain group. The cutoff value for high fall risk in the symptoms of falling factor is set at one point [25], and the corresponding fall prevalence rate is $28 \%$ [26]. The symptoms of falling score for the severe knee pain group in this study exceeded one point (severe unilateral pain, 1.2 points; severe bilateral knee pain, 1.5 points). Taken together, it was found that one out of four persons had a risk of falling.

The disease and physical symptoms score was significantly higher in the mild and severe knee pain groups 
than in the no pain group. Because the presence or absence of lower limb joint pain is included among the items constituting this factor (see Table 1), it is possible that the group difference occurred because of the effect of the answers. However, the disease and physical symptoms score for the severe knee pain group was almost 2 points higher than the score for the no knee pain group. Taken together, elderly individuals with severe knee pain may have other diseases and physical symptoms in addition to knee pain. A decrease in visual and/or auditory acuity and osteoporosis may also have affected this score because patients in the severe knee pain group were older than those in the no knee pain group.

Scores for the environment factor and behavior and character factor were not significantly different among groups. As stated above, subjects in this study were grouped by the degree of the pain and laterality of pain. Our results suggested that knee pain was not related to the above two factors. These items (see Table 1) can be improved by individual awareness. Elderly individuals with knee pain may decrease their fall risk by improving the aspects indicated in the items constituting the environment and behavior and character factors.

The physical function score as well as the total fall risk score was significantly higher in the following order: severe knee pain group, mild knee pain group, and no knee pain group. A decrease in ADL ability is considered to be a major factor underlying falls [3] [4]. In short, it is inferred that physical function is affected by the degree of knee pain [27]. The ability to achieve ADL is improved by a decrease in knee pain and a consequent decrease in fall risk. On the other hand, the symptoms worsen with continuing knee pain and may increase fall risk. It is important to decrease knee pain and improve the ability to achieve ADL independently.

\section{Conclusion}

For elderly females who can achieve ADL independently, the degree of knee pain (mild or severe) has a marked effect on fall risk; however, the laterality of pain (unilateral or bilateral) does not relate to this risk. Elderly individuals with severe knee pain may experience frequent falls, and the risk of falling in these individuals is largely affected by factors such as symptoms of falling, disease and physical symptoms, and physical function. Meanwhile, elderly individuals with mild knee pain and fewer fall experiences may fall by the effects of the above risk factors.

\section{Acknowledgements}

Research funds were not provided by any institution.

\section{References}

[1] Hurley, M.V., Rees, J. and Newham, D.J. (1998) Quadriceps Function, Proprioceptive Acuity and Functional Performance in Healthy Young, Middle-Aged and Elderly Subjects. Age and Ageing, 27, 55-62. http://dx.doi.org/10.1093/ageing/27.1.55

[2] Jochanan, S., Robert, H.R., Aaron, C., Eliana, E.M. and Jeremy, M.J. (2009) Physical Activity, Function, and Longevity among the Very Old. Archives of Internal Medicine, 169, 1476-1483. http://dx.doi.org/10.1001/archinternmed.2009.248

[3] Tinetti, M.E. and Williams, C.S. (1998) The Effect of Falls and Fall Injuries on Functioning in Community-Dwelling Older Persons. The Journal of Gerontology. Series A, Biological Sciences and Medical Sciences, 53, 112-119.

[4] Mary, E. and Tinetti, M.D. (2003) Preventing Falls in Elderly Persons. New England Journal of Medicine, 348, 42-49. http://dx.doi.org/10.1056/NEJMcp020719

[5] Corrigan, D. and Bohannon, R.W. (2001) Relationship between Knee Extension Force and Stand-Up Performance in Community-Dwelling Elderly Women. Archives of Physical Medicine and Rehabilitation, 82, 1666-1672. http://dx.doi.org/10.1053/apmr.2001.26811

[6] Oya, Y., Nakamura, M., Tabata, E., Morizono, R., Mori, S., Komuro, Y. and Horikawa, E. (2008) Fall Risk Assessment and Knee Extensor Muscle Activity in Elderly People. Journal of Geriatrics, 45, 308-314. http://dx.doi.org/10.3143/geriatrics.45.308

[7] Peat, G., McCarney, R. and Croft, P. (2001) Knee Pain and Osteoarthritis in Older Adults: A Review of Community Burden and Current Use of Primary Health Care. Annals of the Rheumatic Diseases, 60, 91-97. http://dx.doi.org/10.1136/ard.60.2.91

[8] American Geriatrics Society, British Geriatrics Society and American Academy of Orthopaedic Surgeons Panel on Falls Prevention. (2001) Guideline for the Prevention of Falls in Older Persons. Journal of the American Geriatrics 
Society, 49, 664-672. http://dx.doi.org/10.1046/j.1532-5415.2001.49115.x

[9] Okanishi, T. and Tsurumi, T. (2001) Physiotherapy of Leg Joint Disorders. Mitsuwa Shoten, Tokyo.

[10] Tennant, A., Fear, J., Pickering, A., Hillman, M., Cutts, A. and Chamberlain, M.A. (1995) Prevalence of Knee Problems in the Population Aged 55 Years and Over: Identifying the Need for Arthroplasty. British Medical Journal, 310, 1291-1293. http://dx.doi.org/10.1136/bmj.310.6990.1291

[11] Al-Zahrani, K.S. and Bakheit, A.M. (2002) A Study of the Gait Characteristics of Patients with Chronic Osteoarthritis of the Knee. Disability and Rehabilitation, 24, 275-280. http://dx.doi.org/10.1080/09638280110087098

[12] Zoltan, B., Robert, P., Arpad, I. and Rita, M.K. (2006) The Influence of Walking Speed on Gait Parameters in Healthy People and in Patients with Osteoarthritis. Knee Surgery, Sports Traumatology, Arthroscopy, 14, 612-622. http://dx.doi.org/10.1007/s00167-005-0005-6

[13] Mc Alindon, T.E., Snow, S., Cooper, C. and Dieppe, P.A. (1992) Patterns of Osteoarthritis of the Knee Joint in the Community: The Importance of the Patellofemoral Joint. Annals of the Rheumatic Diseases, 51, 844-849. http://dx.doi.org/10.1136/ard.51.7.844

[14] Reilly, S.C., Muir, K.R. and Doherty, M. (1996) Screening for Pain in Knee Osteoarthritis: Which Question? Annals of the Rheumatic Diseases, 55, 931-933. http://dx.doi.org/10.1136/ard.55.12.931

[15] Urwin, M., Symmons, D., Allison, T., Brammah, T., Busby, H. and Roxby, M. (1998) Estimating the Burden of Musculoskeletal Disorders in the Community: The Comparative Prevalence of Symptoms at Different Anatomical Sites, and the Relation to Social Deprivation. Annals of the Rheumatic Diseases, 57, 649-655. http://dx.doi.org/10.1136/ard.57.11.649

[16] Astephen, J.L., Deluzio, K.J., Caldwell, G.E. and Dunbar, M.J. (2008) Biomechanical Changes at the Hip, Knee, and Ankle Joints during Gait Are Associated with Knee Osteoarthritis Severity. Journal of Orthopaedic Research, 26, 332341. http://dx.doi.org/10.1002/jor.20496

[17] Zeni, J.A. and Higginson, J.S. (2009) Differences in Gait Parameters between Healthy Subjects and Persons with Moderate and Severe Knee Osteoarthritis: A Result of Altered Walking Speed? Clinical Biomechanics, 24, 372-378. http://dx.doi.org/10.1016/j.clinbiomech.2009.02.001

[18] Oida, Y. and Nakamura, N. (2008) Exercise Learning Support Manual for the Knee Joint Ache Prevention and Reduction. Sunlife Plan, Tokyo.

[19] Dieppe, P.A. and Lohmander, L.S. (2005) Pathogenesis and Management of Pain in Osteoarthritis. The Lancet, 365, 965-973. http://dx.doi.org/10.1016/S0140-6736(05)71086-2

[20] Hashimoto, H., Hanyu, T., Sledge, C.B. and Lingard, E.A. (2003) Validation of a Japanese Patient-Derived Outcome Scale for Assessing Total Knee Arthroplasty: Comparison with Western Ontario and McMaster Universities Osteoarthritis Index (WOMAC). Journal of Orthopaedic Science, 8, 288-293. http://dx.doi.org/10.1007/s10776-002-0629-0

[21] Bellamy, N., Buchanan, W.W., Goldsmith, C.H., Campbell, J. and Stitt, L.W. (1988) Validation Study of WOMAC: A Health Status Instrument for Measuring Clinically Important Patient Relevant Outcomes to Antirheumatic Drug Therapy in Patients with Osteoarthritis of the Hip or Knee. Journal of Rheumatology, 15, 1833-1840.

[22] Demura, S., Sato, S., Yokoya, T. and Sato, T. (2010) Examination of Useful Items for the Assessment of Fall Risk in the Community-Dwelling Elderly Japanese Population. Environmental Health and Preventive Medicine, 15, 169-179.

[23] Sugiura, H. and Demura, S. (2012) The Effects of Knee Joint Pain and Disorders on Knee Extension Strength and Walking Ability in the Female Elderly. Advances in Physical Education, 2, 139-143. http://dx.doi.org/10.4236/ape.2012.24024

[24] Vignon, E., Valat, J.P., Rossignol, M., Avouac, B., Rozenberg, S., Thoumie, P., Avouac, J., Nordin, M. and Hilliquin, P. (2006) Osteoarthritis of the Knee and Hip and Activity: A Systematic International Review and Synthesis (OASIS). Joint Bone Spine, 73, 442-455. http://dx.doi.org/10.1016/j.jbspin.2006.03.001

[25] Demura, S., Sato, S., Shin, S. and Uchiyama, M. (2011) Setting the Criterion for Fall Risk Screening for Healthy Community-Dwelling Elderly. Archives of Gerontology and Geriatrics, 54, 370-373. http://dx.doi.org/10.1016/j.archger.2011.04.010

[26] Sato, S., Demura, S., Shin, S., Sugiura, H. and Uchida, Y. (2012) Assessing a Personal and Population Fall Risk Profile in Japanese Community-Dwelling Elderly. Japan Journal of Test and Evaluation in Health and Physical Education, 11, 49-55.

[27] Sugiura H. and Demura, S. (2013) Effects of Mild and Severe Knee Joint Pain on Various Activities of Daily Living in the Female Elderly. Pain Research and Treatment, 2013, Article ID 989508. http://dx.doi.org/10.1155/2013/989508 
Scientific Research Publishing (SCIRP) is one of the largest Open Access journal publishers. It is currently publishing more than 200 open access, online, peer-reviewed journals covering a wide range of academic disciplines. SCIRP serves the worldwide academic communities and contributes to the progress and application of science with its publication.

Other selected journals from SCIRP are listed as below. Submit your manuscript to us via either submit@scirp.org or Online Submission Portal.
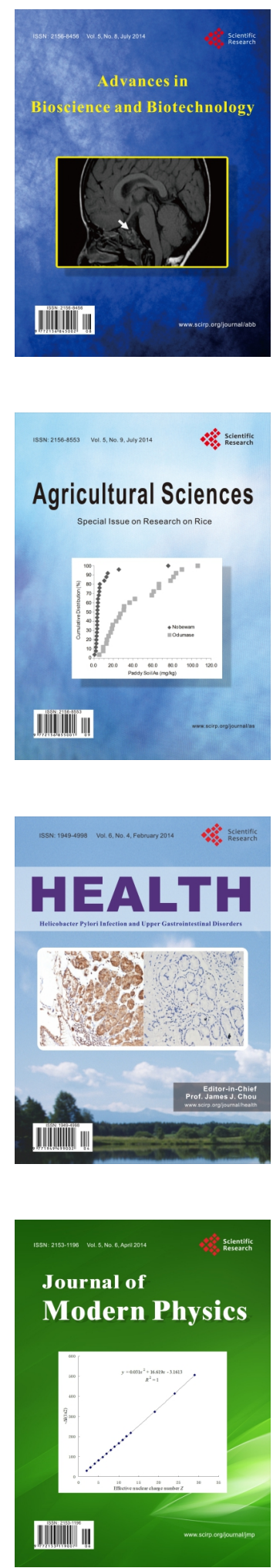
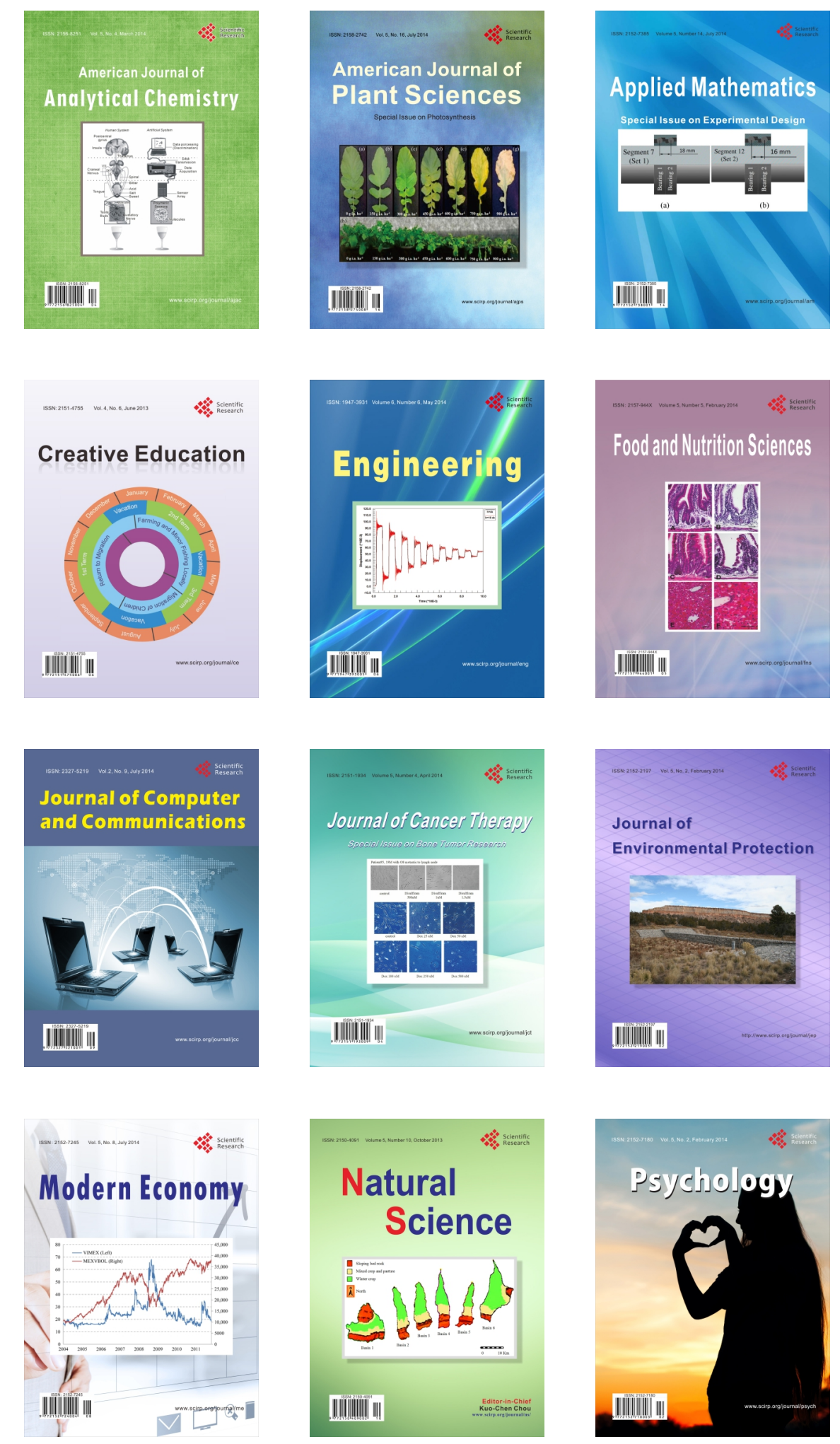